EPJ Web of Conferences 66, 02107 (2014)

DOI: $10.1051 /$ epjconf/ 20146602107

(C) Owned by the authors, published by EDP Sciences, 2014

\title{
Mass Measurement of Short-lived Nuclei at HIRFL-CSR
}

\author{
M. Wang ${ }^{1 a}$, H.S. Xu${ }^{1}$, Y.H. Zhang ${ }^{1}$, X.L. Tu ${ }^{1}$, Yu.A. Litvinov ${ }^{2}$ and CSRe collaboration \\ ${ }^{1}$ Institute of Modern Physics, Chinese Academy of Sciences, Lanzhou 730000, China \\ ${ }^{2}$ GSI Helmholtzzentrum für Schwerionenforschung, Planckstrasse 1, 64291 Darmstadt, Germany
}

\begin{abstract}
Four campaigns of mass measurements for short-lived nuclei have been conducted using an isochronous mass spectrometry (IMS) technique at HIRFL-CSR (Cooler Storage Ring) in Lanzhou. The radioactive nuclei were produced by projectile fragmentation and injected into the experimental storage ring CSRe. Revolution times of the ions stored in the CSRe were measured from which masses of ${ }^{78} \mathrm{Kr},{ }^{58} \mathrm{Ni},{ }^{86} \mathrm{Kr}$ and ${ }^{112} \mathrm{Sn}$ fragments have been determined with a relative uncertainty of about $10^{-6}-10^{-7}$. The experimental results are presented and their impacts on nucleosynthesis in the rp process and nuclear structure are discussed.
\end{abstract}

\section{Introduction}

The mass is a fundamental property of atomic nuclei that reflects the sum of all interactions that hold the nucleons together in the nucleus. Nuclear masses are widely used in basic nuclear physics and astrophysics research, as well as in some other practical applications such as nuclear energy. Although the masses of nuclei along the stability valley are in general well known, more works need to be done for the masses of short-lived nuclei far from the stability. These mass values are urgently demanded to study topics such as nuclear shell evolution far from the stability and to understand the explosive nucleosynthesis processes in astrophysics. While moving far away from the stability, more technical challenges are encountered, due to the very low production rates and the short lives of the nuclei of interest. In recent years, many efforts have been dedicated to mass measurements of short-lived nuclei [1]. The nuclear landscape of known masses have been extended impressively [2], mainly thanks to the developments of Penning traps and the storage rings.

The nuclear storage ring, combined with the fragments separator, is a powerful tool for mass measurement of short-lived nuclei [3]. The CSR (Cooler Storage Ring) is the update project of HIRFL, the heavy ion research facility in Lanzhou. The layout of HIRFL-CSR is shown in figure 1. With the previously existing cyclotron SFC or SSC as the injector, the CSR complex consists a synchrotron CSRm, an experimental ring CSRe and the beam line RIBLL2 (Radioactive Ion Beam Line in Lanzhou) interconnecting these two rings [4]. With the commissioning, the isochronous mass spectrometry was successfully realized at CSRe in the end of 2007 [5], following the pioneer work at GSI-ESR [6]. Afterwards four campaigns of mass measurements of short-lived nuclei have been carried out at the CSR. The experiments and some of the results are described in this contribution.

\footnotetext{
${ }^{\text {a }}$ Corresponding author: wangm@impcas.ac.cn
} 


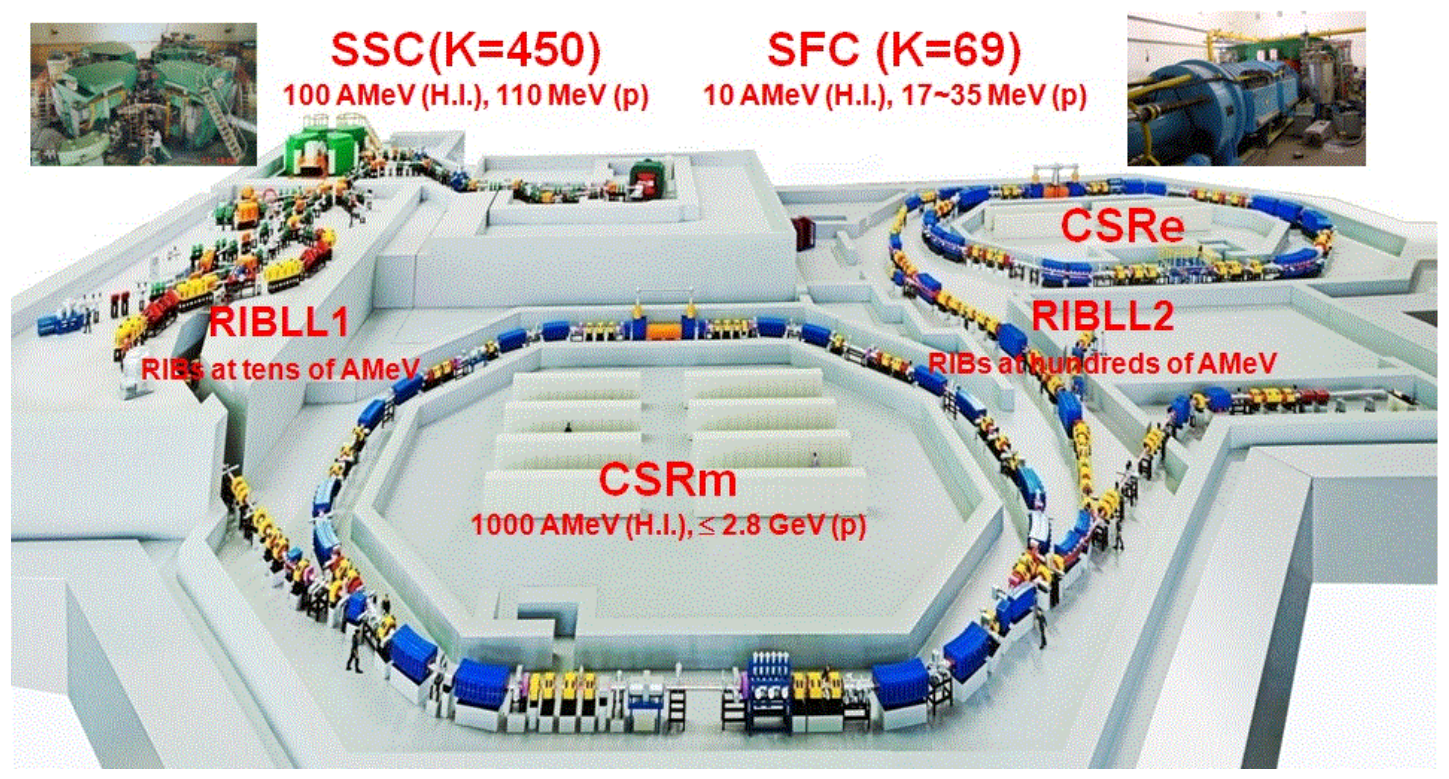

Figure 1. Layout of HIRFL-CSR.

\section{Experiments of mass measurement at CSR}

\subsection{Procedure of experiments}

The nuclei of interest are produced by projectile fragmentation. In the four campaigns of mass measurements that have been performed, the primary beams of ${ }^{78} \mathrm{Kr},{ }^{58} \mathrm{Ni},{ }^{86} \mathrm{Kr}$ and ${ }^{112} \mathrm{Sn}$ were used respectively. The primary beams are accumulated and accelerated at CSRm, and then fast extracted to bombard a beryllium production target at the entrance of RIBLL2. The fragments of interest are selected by the magnetic setting of RIBLL2. The nuclei are injected into the CSRe and then stored there.

The revolution times $\mathrm{T}$ of various stored ions are related (in first order) to their mass-to-charge ratios $\mathrm{m} / \mathrm{q}$ via equation [7]

$$
\frac{\Delta T}{T}=\frac{1 \Delta(m / q)}{\gamma_{t}^{2}(m / q)}-\left(1-\frac{\gamma^{2}}{\gamma_{t}^{2}}\right) \frac{\Delta v}{v}
$$

where $\gamma$ is the relativistic Lorentz factor and $\gamma_{t}$ denotes the transition point of the storage ring. The revolution times of the ions reflect directly $\mathrm{m} / \mathrm{q}$ ratios of the stored ions, if the term containing their velocity spreads, $\Delta v / v$, is minimized. For this purpose, the CSRe was tuned in an isochronous ionoptical mode and the energy of the primary beam was chosen such that $\gamma$ of the ions of interest approximate to $\gamma_{t}$. In this way, the faster particles circulate in the longer orbits and the slower ones in shorter orbits, while the net revolution times are independent of their $\Delta v / v$. In the first three experiments (with ${ }^{78} \mathrm{Kr},{ }^{58} \mathrm{Ni},{ }^{86} \mathrm{Kr}$ as the primary beams), $\gamma_{\mathrm{t}}$ was set to be 1.395 , where the energy of the nuclei of interest is around $367 \mathrm{MeV} / \mathrm{u}$. Thus the energy of the primary beam has to be higher than $460 \mathrm{MeV} / \mathrm{u}$ to produce fragments sufficiently on the production target. In principle, lower $\gamma_{\mathrm{t}}$ corresponds to lower momentum acceptance of the ring. During the experiment with ${ }^{112} \mathrm{Sn}$ beam, the $\gamma_{\mathrm{t}}$ of 1.302 was set, where ${ }^{112} \mathrm{Sn}$ beam of lower than $400 \mathrm{MeV} / \mathrm{u}$ was employed. Optimizing the setting, the momentum acceptance of the ring was around $\pm 0.2 \%$, roughly the same as the previous experiments.

The revolution times were measured using a timing detector [8] equipped with a $19 \mu \mathrm{g} / \mathrm{cm}^{2}$ thin carbon foil of $40 \mathrm{~mm}$ in diameter. Each stored ion passed through the timing detector at every revolution in CSRe. Secondary electrons released from the foil due to the passing ions were guided 
isochronously by perpendicularly arranged electrostatic and magnetic fields to a set of microchannel plates. The time resolution of the detector was about $50 \mathrm{ps}$, and the detection efficiency varied from $20 \%$ to $70 \%$ depending on the ion type and the number of the ions stored. The signals from the detector were sampled with a digital oscilloscope Tektronix DPO 71254 at a sampling rate of $50 \mathrm{GHz}$. The recording time was $200 \mu$ s for each injection, which corresponds to $\sim 320$ revolutions in the first three experiments and $\sim 300$ revolutions in the ${ }^{112} \mathrm{Sn}$ experiment. In principle, nuclei with half-lives down to several tens $\mu$ s can be measured with the IMS, where nuclei can survive long enough to extract the revolution times. Since all of known $\beta$-decaying nuclear states have half-lives longer than 1 ms, most of the nuclear chart can be covered by this method.

\subsection{Data analysis}

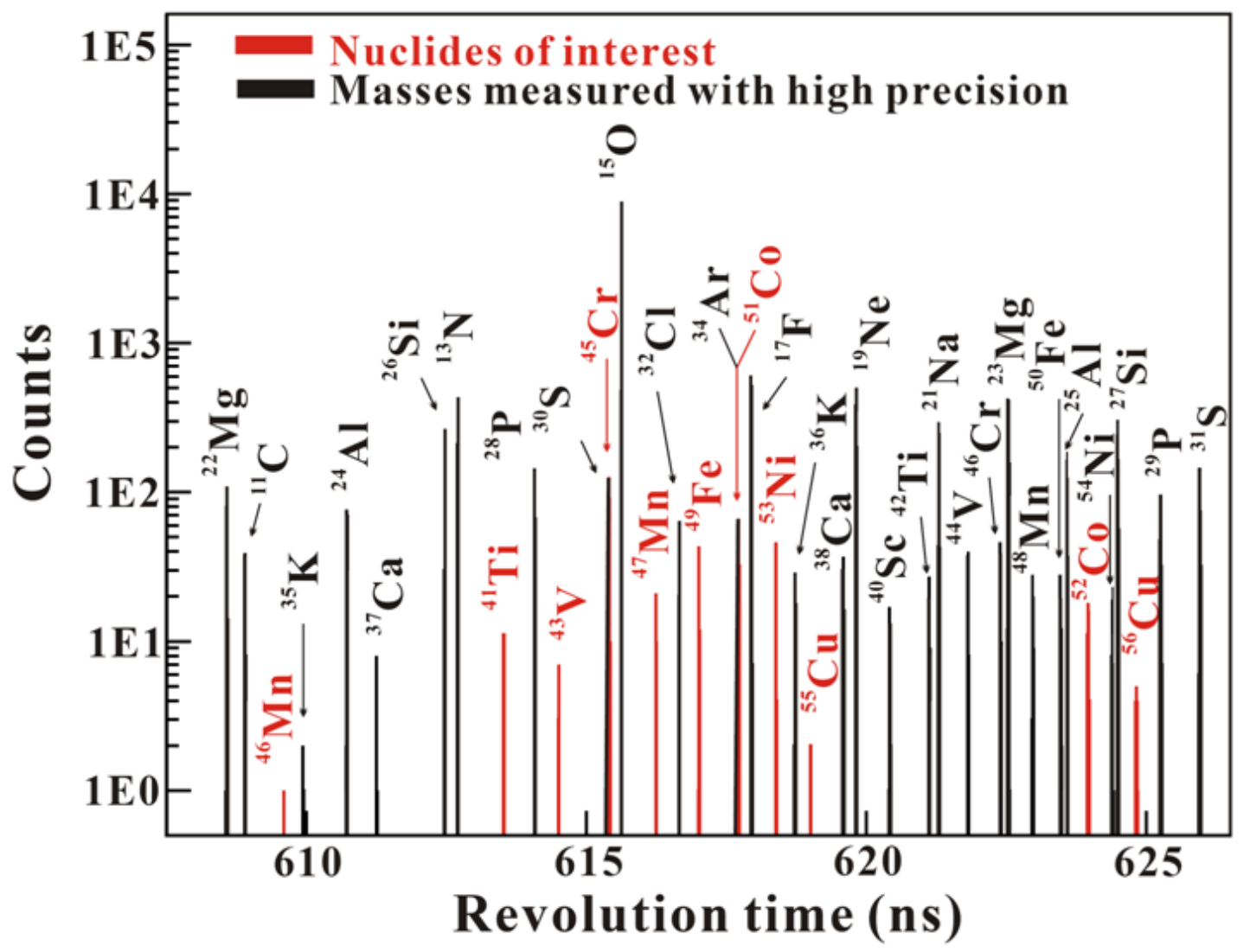

Figure 2. The revolution time spectrum obtained in ${ }^{58} \mathrm{Ni}$ experiment.

Particle identification was realized by comparing the measured revolution-time spectrum with a simulated one. A typical spectrum of the revolution time obtained in ${ }^{58} \mathrm{Ni}$ experiment is given in figure 2. The nuclei with experimentally known masses are used to fit $\mathrm{m} / \mathrm{q}$ versus $\mathrm{T}$ employing a polynomial function, and then the masses of interest are determined. We try to use the lowest possible order to fit the data in order to have a robust fitting and to avoid fitting the random fluctuations. A second order polynomial is chosen in our data analysis. No significant systematic errors are observed in the experiments and the errors for the new masses are dominated by statistical errors. In mass measurements at CSR, a relative uncertainty of about $10^{-6}-10^{-7}$ has been achieved, depending on the statistics of the specific nuclei and the distance of $\gamma$ value of the nuclei to the $\gamma_{t}$ set in experiments.

The resolving power of CSRe mass spectrometry is mainly determined by instabilities of the magnetic fields. These instabilities cause small shifts in the revolution time of the entire spectrum for 
different injections. In order to reduce the effect of these shifts, we developed a novel method in the first experiment (with ${ }^{78} \mathrm{Kr}$ as the primary beam) by using the revolution time of each particle relative to the revolution time of a selected reference nuclide present in the same injection. The details of the method are described in [9]. The resolving power of 170,000 has been achieved with this method. Since not all the nuclei were present in each injection, only $69 \%$ of all data where the reference nuclei were available were used in the mass determination in ${ }^{78} \mathrm{Kr}$ experiment. We are developing new methods by employing all of the available data.

In later experiments, the time stability of the magnetic field was improved to about $10^{-5}$, better than the first experiment by more than one order of magnitude, thanks to an update of the power supply system of CSRe. The data analysis method of using relative revolution frequencies was not necessary. The integral spectra of revolution times were obtained by grouping the injections where the magnetic fields were stable.

\section{Discussion of the experimental results}

In the four experiments, the masses of more than 10 nuclei have been measured for the first time in $\mathrm{CSRe}$, and more than 20 other nuclei have been remeasured with higher accuracy, as shown in figure 3 . Some of the results have been published in scientific journals and more results are coming out. Here we will discuss the physics significance related to some of the results.

Re-measured at CSRe with improved precision

Measured for the first $\mathrm{Co}_{0}$ time at CSRe

$\operatorname{Mg}$

$\begin{array}{lllllllll}10 & 12 & 14 & 16 & 18 & 20 & 22 & 24\end{array}$
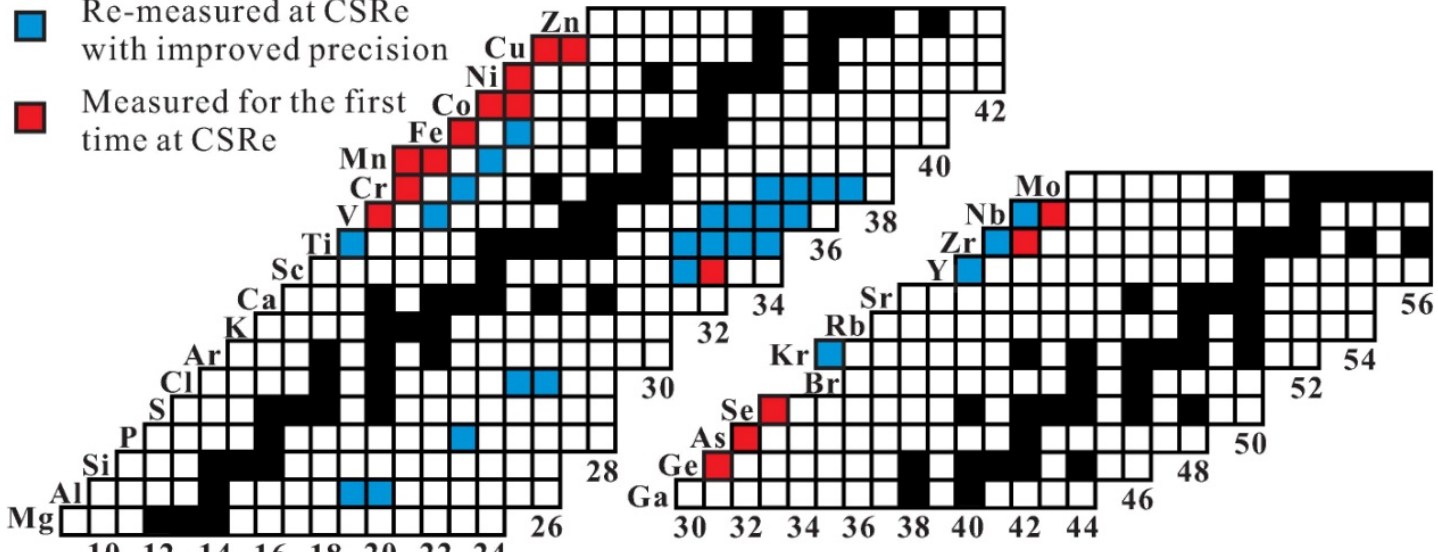

Figure 3. Part of the nuclear chart that has been covered by mass measurements at CSR.

\subsection{Impact on the rp-process}

Some of the nuclei measured at CSR are located on the path of the rapid proton capture (rp) process which is believed to power the type I X-ray bursts. ${ }^{64} \mathrm{Ge}$ is a potentially important waiting point along rp-process because of its long half life. The effective lifetime of ${ }^{64} \mathrm{Ge}$ in the stellar environment is sensitively related to the proton separation energy of ${ }^{65} \mathrm{As}$. By measuring the mass of ${ }^{65} \mathrm{As}$ in our experiment, the proton separation energy for ${ }^{65} \mathrm{As}$ is deduced to be $-90(85) \mathrm{keV}$. X-ray burst model calculations with the new mass excess of ${ }^{65} \mathrm{As}$ suggest that the majority of the reaction flow passes through ${ }^{64} \mathrm{Ge}$ via proton capture, indicating that ${ }^{64} \mathrm{Ge}$ is not a significant rp-process waiting point [10].

The mass of ${ }^{45} \mathrm{Cr}$ nuclide is essential for modeling the astrophysical rp-process concerning the formation of the predicted Ca-Sc cycle in X-ray bursts. The critical branch point in the reaction flow is ${ }^{43} \mathrm{Ti}$. The net proton capture flow at ${ }^{43} \mathrm{Ti}$ is determined by $2 \mathrm{p}$-capture process on ${ }^{43} \mathrm{Ti}$. A low $\mathrm{S}_{\mathrm{p}}\left({ }^{45} \mathrm{Cr}\right)$ however would hamper the proton capture flow at ${ }^{43} \mathrm{Ti}$ because ${ }^{45} \mathrm{Cr}(\gamma, \mathrm{p})$ becomes effective. As a result, a significant $\beta$-decay branch develops at ${ }^{43} \mathrm{Ti}$ driving the reaction flow into ${ }^{43} \mathrm{Sc}$, which has a particularly high $\mathrm{Q}$-value for $(\mathrm{p}, \alpha)$ reactions. The resulting large $(\mathrm{p}, \alpha)$ reaction leads to the formation 
of a Ca-Sc cycle that strongly limits the reaction flow toward heavier elements, leading to a significant increase in burst rise time, a broader main burst, and a more rapid end of nuclear burning. The formation of the Ca-Sc cycle in X-ray bursts depends on the nuclear masses, including the mass of ${ }^{45} \mathrm{Cr}$. With our new measurement of the ${ }^{45} \mathrm{Cr}$ mass we obtain $\mathrm{S}_{\mathrm{p}}\left({ }^{45} \mathrm{Cr}\right)=2.69 \pm 0.13 \mathrm{MeV}$, basically excluding the formation of a significant $\mathrm{Ca}-\mathrm{Sc}$ cycle in X-ray bursts [11].

\subsection{Test of IMME in pf-shell nuclei}

Isospin symmetry is a fundamental concept in nuclear and particle physics. Assuming the two-body nature for any charge dependent effects and the Coulomb force between the nucleons, masses of the $2 \mathrm{~T}+1$ members of an isobaric multiplet are related by the isobaric multiplet mass equation (IMME):

$$
M E\left(A, T, T_{z}\right)=a(A, T)+b(A, T) T_{z}+c(A, T) T_{Z}^{2}
$$

where $\mathrm{a}, \mathrm{b}, \mathrm{c}$ are parameters depending on the atomic mass number $\mathrm{A}$ and the total isospin $\mathrm{T}$. By using ${ }^{58} \mathrm{Ni}$ as the primary beam, the masses of a series of $\mathrm{T}_{\mathrm{z}}=3 / 2$ nuclei from ${ }^{41} \mathrm{Ti}$ through ${ }^{53} \mathrm{Ni}$ were measured. This allowed us for the first time to perform a test of IMME in fp-shell nuclei. We found a breakdown of the quadratic form of IMME for the $A=53(T=3 / 2)$ quartet [12]. The disagreement cannot be explained by either the existing or the new theoretical calculations of isospin mixing. The result will arouse intense interest of nuclear experimentalists in measuring more accurately the masses of relevant nuclei and the excitation energies of the IAS, and inspire nuclear theorists to study the physical causes of isospin symmetry and breaking at the nucleus level.

\section{Perspective}

The isochronous condition is only fulfilled strictly in a small $\mathrm{m} / \mathrm{q}$-range with a storage ring. In real experiments the ring can only be tuned for some specific nuclei, where the resolving power is the highest and it slowly decreases towards both sides of the revolution-time spectrum. To overcome this, it is planned that two time-of-flight detectors are installed in one of the straight sections of the CSRe. In this way, velocities of the individual stored ions can be measured in the ring. The information on the particle velocities should allow us to obtain a high resolving power for the entire revolution-time spectrum, which has an extension of $\Delta(\mathrm{m} / \mathrm{q}) /(\mathrm{m} / \mathrm{q})=10 \%$. The two timing detectors are under test in our laboratory and will be installed in near future. Thus, a larger number of nuclei will be measured or used as references in a single setting of the future RIBLL2-CSRe system.

\section{Acknowledgement}

This work is supported by the Major State Basic Research Development Program of China (973 Program: New physics and technology at the limits of nuclear stability), the NSFC Grants No. 10925526, U1232208, 11035007, 10675147, 10805059, 11135005, and 11075103, the Chinese Academy of Sciences (Grant No. 2009J2-23), the BMBF Grant in the framework of the Internationale Zusammenarbeit in Bildung und Forschung (01DO12012), and the Helmholtz-CAS Joint Research Group HCJRG-108.

\section{References}

1. K. Blaum, J. Dilling, W. Nortershauser, Phys. Scr. T 152, 014017 (2013)

2. G. Audi, M. Wang, A.H. Wapstra, F.G. Kondev, M. MacCornick, X. Xu, B. Pfeiffer, Chin.Phys.C 36, 1287 (2012)

3. Yu.A.Litvinov, H.Geissel, R.Knobel, B.Sun, H.Xu, Acta Phys.Pol. B 41, 511 (2010)

4. W.L.Zhan, H.S.Xu, G.Q.Xiao, J.W.Xia, H.W.Zhao, Y.J.Yuan, Nucl.Phys. A 834, 694c (2010)

5. M.Wang, H.S.Xu, J.W.Xia et al, Int.J.Mod.Phys. E 18, 352 (2009)

6. M. Hausmann et al., Nucl. Instrum. Methods Phys. Res. Sect. A 446, 569 (2000). 
7. M. Hausmann et al., Hyperfine Interact. 132, 289 (2001).

8. B. Mei, X.L. Tu, M. Wang et al., Nucl. Instrum. Methods Phys. Res., Sect. A 624, 109 (2010).

9. X.Tu, M. Wang, Yu. Litvinov et al, Nucl.Instrum.Methods Phys.Res. A 654, 213 (2011)

10. X.L. Tu, H.S. Xu, M. Wang et al., Phys.Rev.Lett. 106, 112501 (2011)

11. X.L. Yan, H.S. Xu, Yu. Litvinov et al., Astrophys.J. 766, L8 (2013)

12. Y.H. Zhang, H.S. Xu, Yu. Litvinov et al., Phys.Rev.Lett. 109, 102501 (2012) 